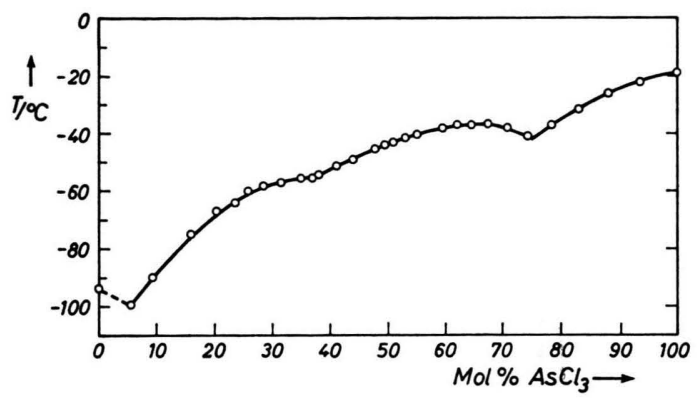

Abb. 6. Liquiduskurve im System Arsentrichlorid-Äthylbenzol, $\mathrm{AsCl}_{3}-\mathrm{C}_{6} \mathrm{H}_{5} \mathrm{C}_{2} \mathrm{H}_{5}$.

nommen (Abbn. 2-6). Die Mischungen neigen zur Unterkühlung, wodurch die Messungen sehr erschwert wurden. Der Verlauf der Liquiduskurven in den Systemen $\mathrm{AsCl}_{3}-p$-Xylol und $\mathrm{AsCl}_{3}-$ Äthylbenzol ist deswegen teilweise unsicher und in den Abbildungen nur gestrichelt eingezeichnet. Im System $\mathrm{AsCl}_{3}-\left(\mathrm{C}_{6} \mathrm{H}_{5}\right)_{2} \mathrm{CH}_{2}$ konnten Mischungen von 45 bis $60 \mathrm{Mol}-\% \mathrm{AsCl}_{3}$ überhaupt nicht zur Kristallisation gebracht werden. Anm. b. d. Korr.: Kürzlich haben W. S. Gretschischkin u. I. A. KJUnzel KQR-Untersuchungen an $\mathrm{AsCl}_{3}$ - und $\mathrm{AsBr}_{3}$-Aromat-Verbindungen veröffentlicht (Tr. Estestv. Nauchn. Inst. pri Permsk. Univ. 11 (4), 9 [1966]; nach C. A. 67, 77742j [1967]).

Für die Durchführung der Schmelzpunktbestimmungen in den Systemen $\mathrm{AsCl}_{3}-\mathrm{ArH}$ sind wir Fräulein K. Shannon zu Dank verpflichtet. Dem Verband der Chemischen Industrie danken wir für eine Beihilfe.

Biosynthese der Mutterkornalkaloide

\title{
Über die Herkunft der Seitenkette im d-Lysergsäure-methylcarbinolamid
}

\author{
D. GRögER *, D. ERGE * und H. G. Floss * * \\ Institut für Biochemie der Pflanzen der DAW, Halle (Saale)* und School of Pharmacy, \\ Purdue University- Lafayette/USA **
}

(Z. Naturforsch. 23 b, 177-180 [1968] ; eingegangen am 24. Mai 1967)

\begin{abstract}
Using a strain of Claviceps paspali Mar 488 it has been shown that alanine-2-C ${ }^{14}$ is incorporated with high efficiency in the carbinol amide moiety of $D$-Lysergic acid- $a$-hydroxyethylamide=II. After feeding of DL-alanine- ${ }^{15} \mathrm{~N}$, DL-aspartic acid- ${ }^{15} \mathrm{~N}$ and L-glutamine-CO $-{ }^{15} \mathrm{NH}_{2}$ the highest specific incorporation rate was observed with DL-alanine ${ }^{15} \mathrm{~N}$. Short term experiments with $\mathrm{D}, \mathrm{L}-$ alanine $-{ }^{15} \mathrm{~N}$ revealed, that the greater part of the ${ }^{15} \mathrm{~N}$-excess is localized in the $\mathrm{N}$-atom of the side-chain of II. The carbinolamide moiety of the isolated alkaloid arises probably from alanine or a closely related compound.
\end{abstract}

Die Biosynthese des Ergolin-Ringsystems - ausgehend von Tryptophan und einer $\mathrm{C}_{5}$-Einheit - sowie die biogenetischen Beziehungen der Clavine und Lysergsäure untereinander sind in verschiedenen Laboratorien intensiv untersucht worden ${ }^{1}$. Wenig ist dagegen über die in vivo-Synthese der Seitenketten säureamidartiger Derivate der Lyserg- bzw. Isolysergsäure vom Peptid-, Alkanolamid-, Amidund Carbinolamid-Typ (I) bekannt. Die Herkunft der Seitenkette im Lysergsäuremethylcarbinolamid = II, des Hauptalkaloids zahlreicher Clavicepspaspali-Stämme, ist von AGURELL ${ }^{2,3}$ untersucht worden. Nach Applikation von Lysergsäure- bzw. Iso-

1 Ubersichten: F. Weygand u. H. G. Floss, Angew. Chem. 75, 783 [1963] ; E. Ramstad u. S. Agurell, Ann. Rev. Plant Physiol. 15, 143 [1964] ; H. Plieninger, Abh. dtsch. Akad. Wiss. Berlin, Kl. Chem. Biol. Geol. 1966, No. 3, 387. lysergsäureamid- $\mathrm{H}^{3}$ (III) an einen Carbinolamidbildenden Stamm ließ sich nur inaktives (II) isolieren. Die Bildung von (II) durch Alkylierung (Acetylierung und nachfolgende Reduktion) der entsprechenden Lysergsäureamide findet offenbar nicht statt. Weiterhin wäre denkbar, daß im Zuge der Synthese von (II) die Amidgruppe des Lysergsäureamids durch Äthylamin ersetzt wird und nachfolgend die Oxydation zum Carbinolamid stattfindet, in Analogie etwa zur Theanin-Biosynthese. Bei Versuchen mit Äthylamin $\cdot{ }^{14} \mathrm{C}$ konnte Agureld ${ }^{2}$ jedoch nur nicht-markierte Alkaloide nachweisen.

2 S. Agurell, Acta pharmac. Suecia 3, 33 [1966].

3 S. Agurell, Acta pharmac. Suecia 3, 71 [1966]. 


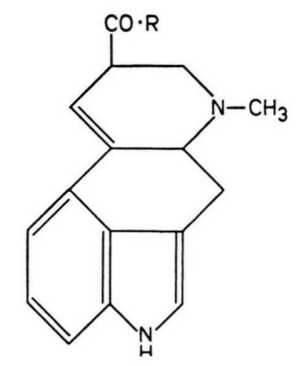

I Allgemeine Formel

Derivate der Lysergsäure. $\mathrm{R}=$ Tripeptid oder einfachere Amid-Derivate.

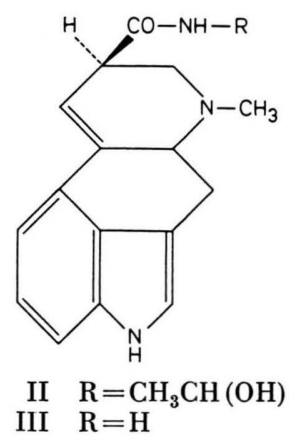

Diese beiden Möglichkeiten scheiden also aus, und Agurell postuliert nun, daß Lysergsäure und Alanin durch eine Peptidbindung zu Lysergylalanin $=(I V)$ verknüpft werden. Reduktion der Carboxylgruppe des Alanins in (IV) würde zum Ergometrin führen; eine Hydroxylierung des Lysergylalanins und nachfolgende Decarboxylierung könnte Carbinolamid ergeben. Castagnoli und Tonolo ${ }^{4}$ applizierten eine Reihe von ${ }^{14} \mathrm{C}$-markierten Verbindungen an $\mathrm{Cl}$. paspali, isolierten (II) und bestimmten die Einbauraten sowie den \%-Gehalt der in der Seitenkette lokalisierten Radioaktivität. Hohe Einbauraten in das Alkaloid ergaben Tryptophan-3.14 C (24\%), Acetat- $1-{ }^{14} \mathrm{C} \quad(10,1 \%)$, Formiat- ${ }^{14} \mathrm{C}$ (6,6\%). Der Carbinolamid-Teil vom (II) war bei diesen Versuchen praktisch inaktiv. Nach Serin-3-14C-Fütterung ließen sich $2,4 \%$ der Radioaktivität des Alkaloids in der Seitenkette nachweisen. Besonders hoch war der Anteil der Radioaktivität im Monoalkylamid-Teil von (II) nach der Applikation von Pyruvat-2-14 C und Alanin-U. ${ }^{14}$ C. Er betrug jeweils $38,9 \%$ und 41,3\% der Gesamt-Radioaktivität. Die Einbauraten dieser Vorstufen in das Lysergsäurecarbinolamid lagen bei $1,6 \%$ und 3,75 Prozent.

4 N. Castagnoli, JR., u. A. Tonolo, IX. Int. Congr. for Microbiology, Moscow 1966, Symposia, p. 31.
Wir haben ähnliche Versuche unter Verwendung von Alanin-2- ${ }^{14} \mathrm{C}$ und verschiedener ${ }^{15} \mathrm{~N}$-markierter Verbindungen durchgeführt. Insbesondere interessierte uns die Herkunft des Stickstoffs im Monoalkylamidteil von (II).

\section{Methodik}

Für diese Untersuchungen wurde der Claviceps paspali-Stamm MAR 488 eingesetzt. Die Kultivierung erfolgte in einem zweistufigen Prozeß. Zur Anzucht des Mycels diente ein corn-steep-Medium (NL 521) ${ }^{5}$. Nach etwa 6-tägiger Kultivation wurde das Mycel abgetrennt, gewaschen und in das eigentliche Fermentationsmedium NL 619 überführt.

Hauptkultur NL 619 Sorbit 50,0 g; Ammonsuccinat $30,0 \mathrm{~g}$; Propylenglycol $20 \mathrm{ml} ; \mathrm{KH}_{2} \mathrm{PO}_{4} 1,0 \mathrm{~g}$; $\mathrm{MgSO}_{4} 0,3 \mathrm{~g}$; Aqua dest. at $1000,0 \mathrm{ml}, p_{\mathrm{H}} 5,4$ eingestellt mit $\mathrm{HCl}$.

Nach 2-3 Tagen erfolgte die Zugabe der markierten Verbindungen. Die Versuchsdauer ist den Tabn. $1-3 \mathrm{zu}$ entnehmen. Nach Abtrennen des Mycels wurde die Nährlösung sofort mit Bicarbonat schwach alkalisch gestellt $\left(p_{\mathrm{H}} 7,8\right)$ und mit Methylenchlorid ausgeschüttelt. Beim Einengen am Rotationsverdampfer fiel Lysergsäure-methylcarbinolamid, welches etwa $2-5 \%$ Ergometrin enthielt, aus. Das so erhaltene Rohalkaloid haben wir aus Chloroform umkristallisiert.

Der Schmelzpunkt, das IR-Spektrum sowie das DCchromatographische Verhalten unseres Präparates stimmten mit dem einer authentischen Probe von Lysergsäure-methylcarbinolamid überein.

Abspaltung von Acetaldehyd: Etwa $20-30 \mathrm{mg}$ des Alkaloids wurden in $6 \mathrm{ml} \mathrm{S}$ ö r e n s e n-Puffer $p_{\mathrm{H}} 7,8$ gelöst und das Ganze unter Durchleiten von Stickstoff 2 Stdn. auf $70-80^{\circ}$ erhitzt. Als Vorlage diente eine 0,5 proz. Lösung von 2.4-Dinitrophenylhydrazin in 2-n. HCl. Das ausgefallene Acetaldehyd-dinitrophenylhydrazon haben wir wiederholt aus Äthanol umkristallisiert, Schmp. $147^{\circ}$.

Hydrolyse von Lysergsäuremethylcarbinolamid: Etwa $50 \mathrm{mg}$ des Alkaloids wurden in wenig Methanol gelöst und anschließend $5 \mathrm{ml}$ 8-proz. $\mathrm{KOH}$ hinzugegeben. Sodann wurde unter Durchleiten eines $\mathrm{N}_{2}$-Stromes $1 \mathrm{Stde}$. auf dem Wasserbad erhitzt. Als Vorlage diente $7 \mathrm{ml}$ 0,1-n. HCl. Nach Abdestillieren blieb $\mathrm{NH}_{4} \mathrm{Cl}$ zurück, welches über $\mathrm{P}_{2} \mathrm{O}_{5}$ getrocknet wurde.

Die ${ }^{15} \mathrm{~N}$-Bestimmung erfolgt nach dem von Munsche ${ }^{6}$ angegebenen Verfahren. Zur Lichtemission wurde der Stickstoff mit dem Hochfrequenzgenerator HF 3 angeregt und das Spectrum mit dem Quarzspektrographen Q 24 aufgenommen. Zur Radioaktivitätsbestimmung diente der Methan-Durchflußzähler der Firma Friesecke und Höpfner.

5 H. G. Floss, H. Günther, D. Gröger u. D. Erge, Z. Naturforschg. 21 b, 128 [1966].

${ }^{6}$ D. Munsche, Kernenergie 8, 32 [1965]. 


\section{Ergebnisse und Diskussion}

Die Einbauraten nach Fütterung von Alanin-2.14 C (Tab. 1) liegen bei etwa 3 Prozent. Beide Versuche zeigen, daß in der Seitenkette des Lysergsäuremethylcarbinolamids ein erheblicher Teil der Radioaktivität lokalisiert ist. Im Vergleich zu den Ergebnissen von Castagnoli und Tonolo ${ }^{4}$, die etwa $40 \%$ der Radioaktivität im Monoalkylamid-Teil von (II) fanden, liegen die Werte unter unseren Bedingungen noch höher. Dies könnte einmal durch das unterschiedliche Pilzmaterial sowie eine andere Kulturtechnik bedingt sein (so ist z. B. die Versuchsdauer von (4) nicht bekannt). Weiterhin ist von (4) Alanin-U-14 C eingesetzt worden, während wir in 2 Stellung markiertes Alanin verfüttert haben. Man darf annehmen, daß im Zuge der CarbinolamidSynthese Alanin decarboxyliert wird und die in der Carboxylgruppe lokalisierten Radioaktivität nicht oder unspezifisch inkorporiert worden ist.

In weiteren Versuchen sollte die Herkunft des Carbinolamid-Stickstoffs geklärt werden. So wurden unter gleichen Versuchsbedingungen (Versuch $36 \mathrm{~B}$, Tab. 2) verschiedene ${ }^{15} \mathrm{~N}$-markierte Verbindungen appliziert und der ${ }^{15} \mathrm{~N}$-Überschuß im Alkaloid und im Monoalkylamid-Teil bestimmt. Gefüttert wurden Alanin $-{ }^{15} \mathrm{~N}$, Aspartat- ${ }^{15} \mathrm{~N}$ sowie Glutamin- $\mathrm{CO}^{15} \mathrm{NH}_{2}$. Die beiden letztgenannten Verbindungen sind an zahl- reichen Transaminierungs-Reaktionen beteiligt und der Amid-N des Glutamins wird leicht auf andere Verbindungen übertragen (z. B. bei der Anthranilsäure-, Purin- und Histidin-Synthese). Ähnliches wäre auch hier denkbar: Eine Reaktion von Lysergsäure und Ammoniak (vom Glutamin) könnte zum Lysergsäureamid (III) führen und dieses eventuell mit einer $\mathrm{C}_{2}$-Einheit zum Carbinolamid umgesetzt werden. Allerdings sprechen die Befunde von Agurell ${ }^{2}$ mit Lysergsäureamid- ${ }^{3} \mathrm{H}$ gegen eine derartige Reaktionsfolge. Auch in unseren Versuchen war der ${ }^{15} \mathrm{~N}$ Überschuß bei Glutamin-CO- ${ }^{15} \mathrm{NH}_{2}$-Fütterung wesentlich geringer als nach Aspartat- und Alaninfütterung (Tab. 2). Die höchste spezifische Einbaurate wurde mit Alanin erzielt. Der Einbau des Aspartat- ${ }^{15} \mathrm{~N}$ könnte durch eine Transaminierungs-Reaktion vielleicht nach dem Schema Aspartat + Pyruvat $\rightarrow$ Alanin erklärt werden. Interessant ist in diesem Zusammenhang der Befund von Castagnoli und Tonolo, die nach Pyruvat-2-14C-Fütterung einen ähnlich großen Einbau im Carbinolamid wie nach Fütterung von Alanin-U.14 C finden konnten.

Vergleicht man in den Versuchen $36 \mathrm{~B}_{5-8}$ (Tab. 2) sowie $35 \mathrm{~K}$ (Tab. 3) den ${ }^{15} \mathrm{~N}-U$ Utberschuß im Alkaloid (3 N-Atome) und im Carbinolamid-Anteil (1 NAtom), so fällt auf, daß die ${ }^{15} \mathrm{~N}$-Anreicherung im Monoalkylamid-Teil nur geringfügig größer ist als die im Alkaloid. Bei einem spezifischen Einbau des

\begin{tabular}{|c|c|c|c|c|c|c|}
\hline Versuch & $\begin{array}{l}\text { Alanin } \\
\text { appliziert }\end{array}$ & $\begin{array}{l}\text { Gebildetes } \\
\text { Alkaloid }\end{array}$ & $\begin{array}{c}\text { Gesamtaktivität } \\
\text { des Alkaloids }\end{array}$ & $\begin{array}{l}\text { Spez. Aktivität } \\
\text { des Alkaloids }\end{array}$ & $\begin{array}{c}\text { Spez. Aktivität } \\
\text { Acetaldehyd-DNP }\end{array}$ & $\begin{array}{c}\text { Radioaktiv. } \\
\text { in der } \\
\text { Seitenkette } \\
{[\%]}\end{array}$ \\
\hline $30 \mathrm{P}$ & $\begin{array}{c}6 \mathrm{mg} \\
6 \cdot 10^{7} \mathrm{Ipm}\end{array}$ & $70 \mathrm{mg}$ & $1,47 \cdot 10^{6} \mathrm{Ipm}$ & $\begin{array}{c}6,55 \cdot 10^{6} \\
\mathrm{Ipm} / \mathrm{mMol}\end{array}$ & $\begin{array}{c}3,7 \cdot 10^{6} \\
\mathrm{Ipm} / \mathrm{mMol}\end{array}$ & 57 \\
\hline $36 \mathrm{Y}$ & $12,2 \mathrm{mg}$ & $205 \mathrm{mg}$ & $3,52 \cdot 10^{6} \mathrm{Ipm}$ & $\begin{array}{c}5,35 \cdot 10^{6} \\
\mathrm{Ipm} / \mathrm{mMol}\end{array}$ & $\begin{array}{c}3,82 \cdot 10^{6} \\
\mathrm{Ipm} / \mathrm{mMol}\end{array}$ & 71 \\
\hline
\end{tabular}

Tab. 1. Einbau von L-Alanin-2-14 C in Lysergsäure-methylcarbinolamid. Spezifische Aktivität des Alanins $8,8 \cdot 10^{8}[\mathrm{Imp} / \mathrm{mM}]$. Versuchszeit 4 Tage.

\begin{tabular}{|c|c|c|c|c|}
\hline Versuchs-Nr. & $\begin{array}{l}\text { Applizierte Verbindungen, in } \\
\text { Klammern }{ }^{15} \mathrm{~N} \text {-Ưberschuß }\end{array}$ & $\begin{array}{c}\text { 15N-Überschuß } \\
\text { im Alkaloid } \\
{[\%]}\end{array}$ & $\begin{array}{c}{ }^{15} \mathrm{~N}-\text { Utberschuß im } \\
\text { Carbinolamid-N } \\
{[\%]}\end{array}$ & $\begin{array}{c}\text { Spez. Einbaurate } \\
\text { im Carbinolamid-N } \\
{[\%]}\end{array}$ \\
\hline 36 B $5-8$ & 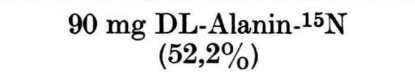 & 0,47 & 0,58 & 1,11 \\
\hline 36 В $9-12$ & $\begin{array}{c}84 \mathrm{mg} \text { DL-Asparaginsäure-15 } \mathrm{N} \\
(40 \%)\end{array}$ & 0,26 & 0,33 & 0,82 \\
\hline 36 В $13-16$ & $80 \mathrm{mg}$ L-Glutamin-CO-15 $\mathrm{NH}_{2}$ & 0,24 & 0,18 & 0,35 \\
\hline
\end{tabular}

Tab. 2. Applikation verschiedener ${ }^{15} \mathrm{~N}$-markierter Verbindungen an $\mathrm{Cl}$. paspali. Für jeden Versuch wurden 4 100-ml-Schüttelkulturen eingesetzt. Der Gesamtalkaloidgehalt betrug $\sim 350 \mathrm{mg}$. Versuchsdauer: 4 Tage. 


\begin{tabular}{cccccc}
\hline Versuchs-Nr. & Versuchsdauer & $\begin{array}{c}15 \text {-Utberschuß } \\
\text { im Alkaloid } \\
{[\%]}\end{array}$ & $\begin{array}{c}15 \text { N-Utberschuß } \\
\text { im Carbinolamid-N } \\
{[\%]}\end{array}$ & $\begin{array}{c}\text { Spez. Einbaurate } \\
\text { im Alkaloid } \\
{[\%]}\end{array}$ & $\begin{array}{c}\text { Spez. Einbaurate } \\
\text { im Carbinolamid-N } \\
{[\%]}\end{array}$ \\
\hline 36 Y 3-4 & $8^{\text {h }}$ & 0,50 & 1,03 & 0,96 & 1,98 \\
36 Q 1-4 & $22^{\mathrm{h}}$ & 0,82 & 1,49 & 1,57 & 2,88 \\
36 Q 5-8 & $48^{\mathrm{h}}$ & 0,60 & 0,97 & 1,15 & 1,9 \\
$35 \mathrm{~K}$ & 4 Tage & 0,48 & 0,53 & 0,92 & 1,02 \\
\hline
\end{tabular}

Tab. 3. Einbau von DL-Alanin ${ }^{15} \mathrm{~N}$ in Lysergsäure-methylcarbinolamid. Es wurden jeweils $80 \mathrm{mg}$ an 2 bzw. 4100 -ml-Kulturen appliziert. Der ${ }^{15} \mathrm{~N}$-Uberschuß im Alanin betrug 52,2 Prozent.

Alanin $-{ }^{15} \mathrm{~N}$ in die Seitenkette des Alkaloids müßten sich die Werte $\left({ }^{15} \mathrm{~N}\right.$-Überschuß) Carbinolamid-N : Alkaloid-N wie $3: 1$ verhalten. Das ist aber nicht der Fall. Daraus geht hervor, daß bei Alanin-Fütterung (Versuchsdauer 4 Tage) auch in den beiden N-Atomen des Ergolins sich ein beträchtlicher Teil des ${ }^{15} \mathrm{~N}$ befinden muß. Offenbar wird ein großer Teil des Alanin-Stickstoffs schnell durch Transaminierungs-Reaktionen in den allgemeinen Stickstoffpool eingeschleust und von dort der Ergolin-Synthese zugeführt. Wir haben deshalb Kurzzeitversuche unternommen und nach der Applikation von Alanin${ }^{15} \mathrm{~N}$ in Intervallen von 8,22 und $48 \mathrm{Stdn}$. (Tab. 3) geerntet. Verläuft der Einbau von Alanin in die Seitenkette schneller als die Transaminierung und der nachfolgende Einbau des Stickstoffs in das Ergolin-System, so sollte der ${ }^{15} \mathrm{~N}$-Überschuß im Carbinol- amid größer als im Alkaloid sein. Wie die Tab. 3 zeigt, ist diese Annahme richtig, da bei den kurzen Versuchszeiten sich die ${ }^{15} \mathrm{~N}$-Werte Carbinolamid : Alkaloid etwa wie $2: 1$ verhalten. Das theoretisch zu erwartende Verhältnis $3: 1$ ließ sich nicht erreichen, da Transaminierungs-Reaktionen nicht verhindert werden können. Auf Grund der Versuche mit Alanin${ }^{15} \mathrm{~N}$ und Alanin- $2 \cdot{ }^{14} \mathrm{C}$ darf man annehmen, daß diese Aminosäure am Aufbau der Seitenkette des Lysergsäurecarbinolamids beteiligt ist. Ob Lysergylalanin dabei als Zwischenstufe durchlaufen wird und in welcher Weise die Hydroxylierung erfolgt, muß noch geprüft werden.

Wir danken Herrn Professor Dr. Arcamone, Mailand, Farmitalia, für die Úberlassung einer Probe d-Lysergsäure-methylcarbinolamid. 